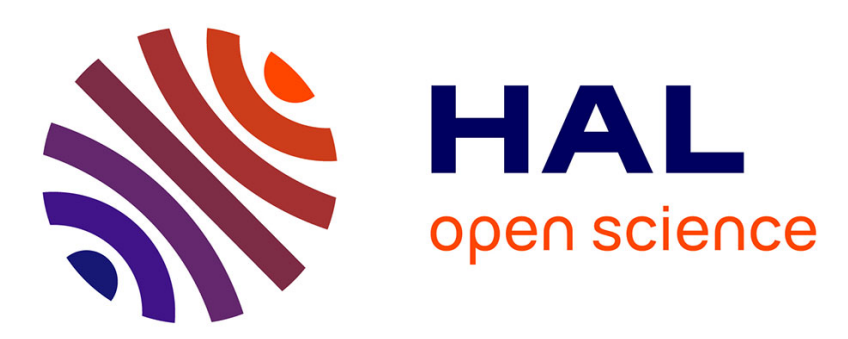

\title{
Application of convergent-beam electron diffraction to the determination of grain boundary structures
}

\author{
F.W. Schapink
}

\section{To cite this version:}

F.W. Schapink. Application of convergent-beam electron diffraction to the determination of grain boundary structures. Revue de Physique Appliquée, 1986, 21 (12), pp.747-755. 10.1051/rphysap:019860021012074700 . jpa-00245496

\section{HAL Id: jpa-00245496 https://hal.science/jpa-00245496}

Submitted on 1 Jan 1986

HAL is a multi-disciplinary open access archive for the deposit and dissemination of scientific research documents, whether they are published or not. The documents may come from teaching and research institutions in France or abroad, or from public or private research centers.
L'archive ouverte pluridisciplinaire HAL, est destinée au dépôt et à la diffusion de documents scientifiques de niveau recherche, publiés ou non, émanant des établissements d'enseignement et de recherche français ou étrangers, des laboratoires publics ou privés. 


\title{
REVUE DE PHYSIQUE APPLIQUÉE
}

\section{Application of convergent-beam electron diffraction to the determination of grain boundary structures}

\author{
F. W. Schapink \\ Laboratory of Metallurgy, Delft University of Technology, Rotterdamseweg 137, 2628 AL Delft, \\ The Netherlands
}

(Reçu le 16 juillet 1986, accepté le 2 septembre 1986)

\begin{abstract}
Résumé. - La diffraction en faisceau convergent (DFC) a été appliquée à la détermination des structures de joints de grains placés horizontalement et verticalement dans une lame mince. D'abord, on établit la relation entre symétrie du bicristal et symétrie du diagramme DFC pour les deux types de joints. Les résultats sont ensuite appliqués pour déterminer la structure de joints de macle dans Au et Si. Les diagrammes DFC de bicristaux maclés sont comparés avec des diagrammes calculés pour un joint de macle horizontal dans $\mathrm{Au}$ et $\mathrm{Si}$. Finalement, la symétrie des diagrammes DFC pour des joints de macle verticaux dans Si est analysée ; elle varie selon que l'on emploie un faisceau incident statique ou oscillant.
\end{abstract}

\begin{abstract}
Convergent-beam electron diffraction (CBED) has been applied in grain boundary structure determination, both for horizontal and vertical boundaries in thin specimens. First the relation between bicrystal symmetry and CBED pattern symmetry is developed for the two types of boundaries. These results are subsequently employed for a structure determination of twin boundaries in $\mathrm{Au}$ and $\mathrm{Si}$. The CBED patterns from twinned bicrystals obtained experimentally are compared with computer-simulated patterns, for the case of a horizontal twin boundary in Au and Si. Finally the symmetry of CBED patterns from vertical twin boundaries in $\mathrm{Si}$ is analysed and found to depend on whether a static incident beam or a rocking-beam geometry is employed.
\end{abstract}

\section{Introduction.}

Conventional electron diffraction patterns have been widely employed for the study of grain boundary structures, primarily in the search for diffraction effects which can be uniquely associated with particular boundary structures. Although this is a very useful method, it suffers from limitations such as the difficulty of obtaining intensities. In the field of crystal structure determination, for example, attempts to deduce crystal symmetries from spot patterns have sometimes resulted in erroneous conclusions. Fortunately, in the area of crystal symmetry determination the technique of convergent-beam electron diffraction (CBED), in which a cone of incident beam directions is employed instead of a single parallel beam, has been shown to be a very valuable tool for the determination of crystal point and space groups $[1,2,3]$. Using this technique it is possible to deduce crystal symmetry from the symmetry of convergent-beam zone-axis patterns (ZAP's) taken from suitable thin specimens.
In this paper the application of CBED to bicrystal specimens is discussed. We consider a particular class of bicrystals, for which the associated dichromatic pattern (i.e. the superimposed lattices or structures of both crystals) has 3-D translation symmetry. The grain boundary in such a bicrystal is necessarily of the coincidence type, and the bicrystal symmetry depends on the state of translation existing at the boundary [4, 5]. Hence by determining bicrystal symmetry from the symmetry of suitable CBED patterns, conclusions about the state of translation at the boundary can be drawn. This is the subject of this paper, and we will consider separately the symmetry properties of CBED patterns from bicrystal specimens with an interface parallel to the specimen surface and perpendicular to the surface, respectively.

\section{CBED pattern symmetry from bicrystals.}

2.1 HORIZONTAL BOUNDARY. - In this section we consider the symmetry of CBED patterns from 


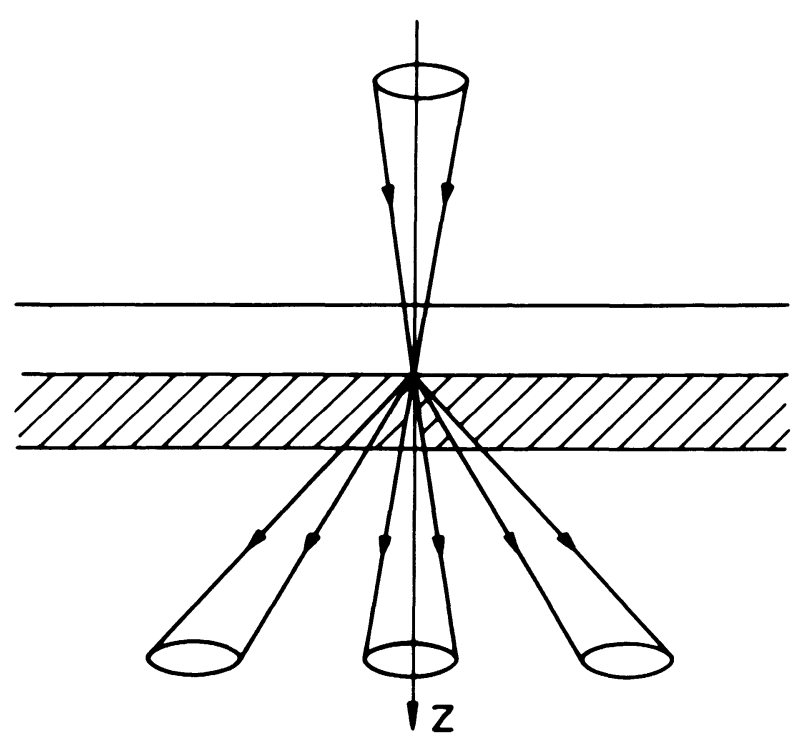

Fig. 1. - Schematic representation of a bicrystal with a horizontal boundary. Incident electron beam along the $z$ axis.

bicrystals with the boundary located at the (ideal) mid-plane position in the specimen, as illustrated in figure 1 . For this purpose the symmetry of a bicrystal specimen should first be classified, and this is done using the concept of the dichromatic pattern (dcp) associated with the bicrystal, as discussed recently by several authors $[6,7,8,9]$. Briefly, the space group of the dcp contains two kinds of symmetry elements. These are : (i) ordinary symmetry elements, which transform the crystals into themselves, and (ii) antisymmetry elements (also called coloured symmetry elements) which interchange the two crystal species in the dcp. Here we consider the case where the translation subgroup of the space group of the dcp defines a 3-D lattice, i.e. there exists a 3-D coincidence lattice in the bicrystal. The problem then is to find the correlation between the dcp symmetry and the CBED pattern symmetry with the cone of incident beams along the specimen normal of figure 1. This implies correlating the possible point groups of a dcp with the 31 diffraction groups necessary for characterizing the symmetry of CBED patterns [1]. The symmetry of the dcp is described by one of the Shubnikov space groups and its point group by one of the 122 possible Shubnikov point groups. Among these 122 groups there are 32 grey groups which are useful to describe stacking-fault or inversion boundaries and will not be considered here. In addition there are 32 monochromatic groups relevant for the description of interphase boundaries which will also be left out of consideration. It remains to consider 58 dichromatic point groups and their relation to the diffraction groups, in order to characterize dcp symmetry from suitable CBED patterns.

From the point of view of electron diffraction from a thin bicrystal specimen one would expect the bicrystal symmetry to be the determining factor instead of the dcp symmetry. This is because the electrons, in travelling through a specimen of the

type shown in figure 1, see the bicrystal as consisting of physically separated crystals. As explained by Pond and Bollmann [6] bicrystal symmetry is obtained from the dcp symmetry by cross-sectioning the dcp on the boundary plane and retaining the sites of one crystal only on either side of the boundary plane. The remaining bicrystal symmetry elements form a subgroup of the original dichromatic point group. Although it is true that bicrystal symmetry determines the CBED pattern symmetry, it will be shown that nevertheless additional symmetry information may be obtained from a consideration of the full dcp symmetry in a number of cases. However, for simplicity we now investigate first the relation between bicrystal symmetry and CBED pattern symmetry, for a bicrystal specimen with the interface coinciding with the specimen mid-plane (Fig. 1).

To find the symmetry relations in a diffraction pattern taken from a thin bicrystal specimen, it is useful to distinguish between horizontal (i.e. parallel to the surface) symmetry elements and vertical symmetry elements existing in a bicrystal point group. A vertical symmetry element enters directly in the diffraction group characterizing the CBED pattern symmetry, whereas a horizontal symmetry element transforms the normal component of the incident beam to its opposite, i.e. transforms $z$ into $-z$ in figure 1. Such an operation therefore does not directly produce symmetry in the diffraction pattern. However, because of the existence of the theorem of reciprocity in electron diffraction these operators lead to symmetry elements in an indirect way and are, as customary, indicated by a subscript $\mathrm{R}$ (for Reciprocity) in the diffraction group [1]. Table I classifies the various possible horizontal and vertical symmetry elements that may exist in a bicrystal layer point group. Ordinary rotation axes and mirror planes are indicated by their standard symbols, whereas primed symmetry elements refer to antisymmetry (coloured) operators, i.e. those operators that interchange both crystals. Note that the coloured rotary-inversion axes $\left(\overline{3}^{\prime}, \overline{4}^{\prime}\right.$ and $\left.\overline{6}^{\prime}\right)$ contain both a vertical component (the rotation operation) and a horizontal component (the operation of antiinversion). The orientations of the symmetry ele-

Table I. - Orientation of symmetry elements in a layer point group associated with the specimen in figure 1 .

Symmetry element of point group

\section{CBED symmetry}

I. Vertical $2,3,4,6$ $\mathrm{m}$

II. Horizontal $\overline{1}^{\prime}, 2^{\prime}$ $\mathrm{m}^{\prime}$

III. Vertical $\overline{3}^{\prime}, \overline{4}^{\prime}, \overline{6}^{\prime}$
Direct symmetry; Operators leave bicrystal and diffracted beams unchanged.

Indirect symmetry; Operators invert bicrystal specimen and introduce symmetry through reciprocity.

Operators combine direct and indirect symmetries. 
ments in table I satisfy the condition that anti-symmetry elements are parallel to the grain boundary, whereas ordinary symmetry elements are perpendicular to the boundary. For a more detailed consideration of the incorporation of various specimen symmetry elements into the diffraction group the reader is referred to the original papers $[1,4,5]$. Here we only consider the final result summarized in table II, where the relation is presented between the 31 bicrystal layer point groups and the 31 diffraction groups originally introduced by Buxton et al. [1]. For ordinary grain boundaries the centro-antisymmetric point groups in table II do not occur, since a centre of antisymmetry implies a coincidence lattice with $\Sigma=1$ ( $\Sigma$ being the reciprocal density of coincidence sites). However, these point groups are relevant for interfaces in crystal structures having a point group which is a subgroup of the point group of the lattice. The one-to-one correspondence between the layer groups and the diffraction groups in table II is a

Table II (from Ref. [5]). - Relation between permissible bicrystal layer point groups and diffraction groups for the specimen and incident beam geometry of figure 1 .

Bicrystal point group ordinary antisymmetric

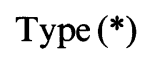

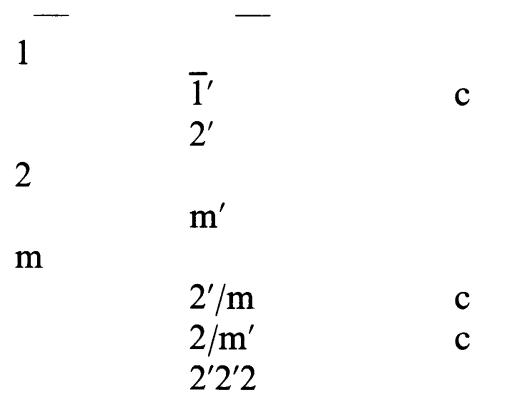

$\mathrm{mm} 2$

$\mathrm{m}^{\prime} \mathrm{m} 2^{\prime}$

$\mathrm{m}^{\prime} \mathrm{mm}$

4

$\overline{4}$

$4 / \mathrm{m}^{\prime}$

$42^{\prime} 2^{\prime}$

$4 \mathrm{~mm}$

3

$\begin{array}{ll}\overline{3} & \mathrm{c} \\ 32^{\prime} & \end{array}$

$3 \mathrm{~m}$

$\overline{3}^{\prime} \mathrm{m}$

6

\section{$\overline{6}$}

$6 / \mathrm{m}^{\prime}$

$62^{\prime} 2^{\prime}$.

$6 \mathrm{~mm}$

$\begin{array}{lll}\overline{6}^{\prime} \mathrm{m} 2^{\prime} & & 6 \mathrm{~mm} \\ 6 / \mathrm{m}^{\prime} \mathrm{mm} & \mathrm{c} & 3 \mathrm{~m} 1_{\mathrm{R}} \\ & & 6 \mathrm{~mm} 1_{\mathrm{R}}\end{array}$

$\left(^{*}\right) \mathrm{c}=$ centro-antisymmetric. consequence of the isomorphism of both types of groups, which in turn is caused by the fact that the anti-symmetry operations in a two-sided layer are equivalent to the reciprocity operation in electron diffraction. This can also be seen from table II if one notes that horizontal symmetry elements in a layer group invariably lead to a diffraction group containing an element with the symbol R (e.g. $2^{\prime}$ leads to $\mathrm{m}_{\mathrm{R}}$ in $\mathrm{Tab}$. II).

It appears useful to illustrate the general principles outlined above with an example. We take the simple case of a (111) coherent twin boundary in either the f.c.c. lattice or the diamond structure. The dichromatic point group is $61 / \mathrm{m}^{\prime} \mathrm{m} / \mathrm{m}$ with the hexad axis parallel to the common [111] axis of matrix and twin (see Fig. 2). If a thin bicrystal is prepared with [111] surface normal for taking a convergent-beam pattern, the dichromatic group has to be sectioned on (111), leaving only an ordinary triad axis, the three ordinary vertical mirror planes, the horizontal $\mathrm{m}^{\prime}$ (the twin boundary plane) and three horizontal $2^{\prime}$ axes making up the layer point group $\overline{6}$ 'm2' (Fig. 2). According to table II the diffraction group of such a twinned crystal specimen is $3 \mathrm{~m} 1_{\mathrm{R}}$, which can be determined from CBED patterns in the [111] orientation (see Sect. 3).

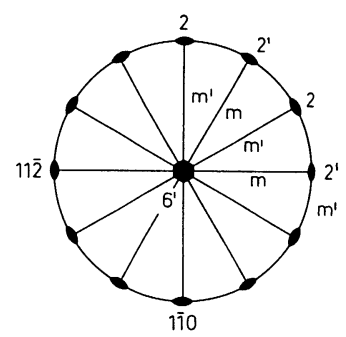

(a)

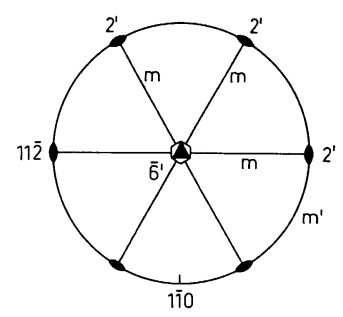

(b)
Fig. 2. - Stereographic projection along [111] of the dichromatic point groups $6^{\prime} / \mathrm{m}^{\prime} \mathrm{m} \prime \mathrm{m}$ and $\overline{6}^{\prime} \mathrm{m} 2^{\prime}$, shown in (a) and (b) respectively. The indices refer to the coordinate system of one of the crystals.

The limitations of this approach to bicrystal symmetry become clear if we consider the effect of a rigid relative translation of the crystals along the boundary normal (i.e. expansion or contraction of the specimen). Such a translation has no effect on bicrystal symmetry as it leaves all symmetry operations of the layer group $\overline{6}^{\prime} \mathrm{m} 2^{\prime}$ unchanged. Hence, one could think that $z$-translations cannot be detected from the symmetry of CBED patterns. This, however, it not true in general as the following considerations show.

The dichromatic point group of the twin is altered from $6^{\prime} / \mathrm{m}^{\prime} \mathrm{m}^{\prime} \mathrm{m}$ to $\overline{6}^{\prime} \mathrm{m} 2^{\prime}$ by a $z$-translation $[10]$, because the vertical anti-mirror planes are destroyed (see Fig. 2). This suggests an investigation of the relation between the 58 possible dichromatic point groups and the diffraction groups, which has been carried out in references [4] and [5]. This investigation, in itself, does not yield any additional informa- 
tion ; for example, the dichromatic groups in figure 2 both lead to diffraction group $3 \mathrm{~m}_{\mathrm{R}}$, as expected. However, consider now what happens if the projection approximation in electron diffraction holds, i.e. if only scattering from the zero-order Laue zone occurs. In single crystals this simply means the addition of a horizontal mirror plane to the specimen point group [1]. However, this is not the case for bicrystals of the type shown in figure 1 . It should be realized that in the projection approximation for bicrystals the distinction between ordinary and antisymmetry elements disappears ; consequently dichromatic point group $6^{\prime} / \mathrm{m}^{\prime} \mathrm{m}^{\prime} \mathrm{m}$ becomes $6 / \mathrm{mmm}$, whereas $\overline{6}$ ' $\mathrm{m} 2^{\prime}$ becomes $\overline{6} \mathrm{~m} 2$. Thus, in the projection approximation a twinned crystal has point group $6 / \mathrm{mmm}$ for zero $z$-translation, whereas the point group is $\overline{6} \mathrm{~m} 2$ for a finite $z$-translation. These two point groups lead to different diffraction groups $\left(6 m m 1_{R}\right.$ and $3 m 1_{R}$ respectively) and can thus be distinguished if 2-D diffraction information in the CBED pattern is available (see Sect. 3).

The example of the twin boundary shows that the most detailed symmetry information is available if both 2-D and 3-D diffraction is present in the CB diffraction pattern. Fortunately the area close to the (000) disc frequently satisfies the conditions of the projection approximation (absence of higher order Laue zone effects) and should therefore be used in obtaining maximum symmetry information. For this purpose a table giving the relation between dichromatic point groups and diffraction groups has been extended to all projection diffraction groups in references [4] and [5].

2.2 VERTICAL BOUNDARY. - Recently we have extended the considerations of the previous section to the case of a thin bicrystal specimen with a vertical boundary [11] (Fig. 3). This boundary orientation

(a)

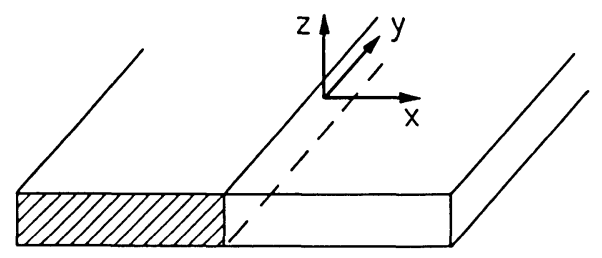

(b)

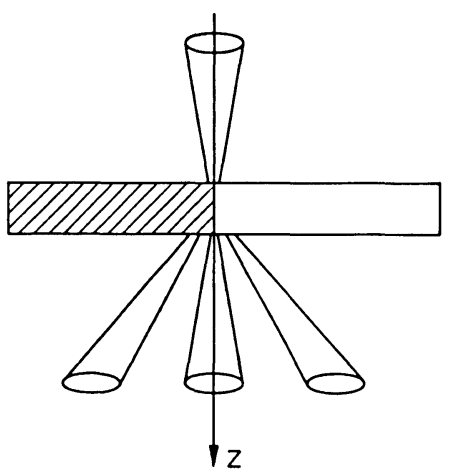

Fig. 3. - Schematic diagram of a bicrystal specimen with a vertical boundary (a) and the geometry of electron diffraction (b). occurs frequently in thin polycrystalline foils and it is therefore important to analyse the symmetry in some detail. Bicrystal symmetry in this case is derived from the dcp symmetry in the same way as before, i.e. after sectioning on the boundary plane and retaining crystal sites of one crystal only on either side of the boundary. The principal difference with the horizontal boundary is, that a thin bicrystal specimen with a vertical boundary should be treated as a bicrystal band, i.e. we have 1-D translation symmetry in the interface [7]. This circumstance simplifies the discussion of possible symmetries considerably, since there are only 12 permissible point groups of bicrystal bands containing the symmetry elements $1, \overline{1}^{\prime}, 2,2^{\prime}, \mathrm{m}$ and $\mathrm{m}^{\prime}$ (where the prime again denotes an antisymmetry element).

In deriving the symmetries of electron diffraction patterns (the diffraction groups) from the bicrystal band point groups the same procedure as in the previous section is followed. That is, a distinction is made between vertical specimen symmetry elements occurring directly in the diffraction group, and horizontal elements that occur in the diffraction group by virtue of the reciprocity theorem.

Table III summarizes the possible horizontal and vertical symmetry elements and table IV presents

Table III. - Possible orientation of symmetry elements in a bicrystal specimen of the type shown in figure 3.

$$
\begin{array}{ll}
\multicolumn{1}{c}{\text { Vertical }} & \multicolumn{1}{c}{\text { Horizontal }} \\
\mathrm{m}(y=\text { Cst.) } & \mathrm{m}(z=0) \\
\mathrm{m}^{\prime}(x=0) & 2(/ / x \text {-axis }) \\
2^{\prime} \text { (along } z \text {-axis) } & 2^{\prime} \text { (along } y \text {-axis) }
\end{array}
$$

Table IV. - Relation between bicrystal band point groups and diffraction groups for a vertical boundary.

$\begin{array}{llcl}\begin{array}{c}\text { Bicrystal point group } \\ \text { ordinary antisymmetric }\end{array} & \begin{array}{c}\text { Type }\left(^{*}\right) \\ 1\end{array} & - & \begin{array}{l}\text { Diffraction } \\ \text { group }(* *)\end{array} \\ & \overline{1}^{\prime} & & \end{array}$

$\left({ }^{*}\right) \mathrm{c}=$ centro-antisymmetric.

$(* *)$ For a given point group different diffraction groups occur for different incident beam directions in the plane $x=0$ (Fig. 3). 
the relation between the bicrystal band point groups and the possible diffraction groups for different orientations of the incident beam. From table IV, the specimen point group cannot in general be determined uniquely from a single pattern ; for this purpose a number of patterns are required. It should be stressed that in this case, contrary to the situation for a horizontal boundary, the effect of making the projection approximation is similar to what happens in a single crystal. That is, the validity of this approximation adds a horizontal mirror plane to the specimen, leading to one of the 4 diffraction groups containing the symbol $1_{R}$.

Let us illustrate these general statements with the example of a vertical coherent (111) twin boundary between f.c.c. crystals. For zero translation the sectioning procedure transforms the dcp symmetry $6^{\prime} / \mathrm{m}^{\prime} \mathrm{m}$ 'm into bicrystal point group $\mathrm{m}^{\prime} \mathrm{m} 2^{\prime}$, where $\mathrm{m}^{\prime}$ coincides with the twin plane and $\mathrm{m}$ is produced by the common $(2 \overline{2} 0)$ planes of the bicrystal. All other symmetry elements are lost in the sectioning procedure. According to table IV, the possible diffraction groups are $2 \mathrm{~mm}$ (incident beam parallel to $[11 \overline{2}]$ ), $\mathrm{m} 1_{\mathrm{R}}$ (incident beam parallel to [1]0]) or $\mathrm{m}$ (incident beam parallel to $[h k l]$ where $h+k+l=0)$. These symmetries also hold for a (111) twin boundary with a translation normal to the twin plane, since such a translation leaves the point group $\mathrm{m}$ 'm2' invariant.

\section{Experimental results.}

In this section we will survey some experimental results on horizontal twin boundaries in Au crystals [4], as well as report on some very recent results concerning an inclined coherent twin boundary in silicon. Subsequently some results relevant to the symmetry determination of vertical coherent twin boundaries. in Si crystals will be discussed.

All experimental results reported in this paper were obtained with a Philips EM400T electron microscope operated in the STEM mode at variable voltage, mostly at $100 \mathrm{kV}$. Care was taken to avoid areas in the specimens in which local defects could have altered the pattern symmetry. In order to reduce diffuse scattering the specimens were cooled to liquid nitrogen temperature in a double-tilt cooling stage. Figure 4 shows a CBED pattern of a thin $\mathrm{Au}$ bicrystal with a horizontal coherent (111) twin boundary, taken along the common [111] axis. As explained in more detail elsewhere [4], the symmetry of this pattern corresponds to diffraction group $3 \mathrm{~m} 1_{\mathrm{R}}$, which leads to $6 \mathrm{~mm}$ symmetry in the central disc and $3 \mathrm{~m}$ symmetry for the whole pattern. As was argued in section 2.1 , diffraction group $3 \mathrm{~m}_{\mathrm{R}}$ for the CBED pattern could have originated from dichromatic point groups $\overline{6}^{\prime} \mathrm{m} 2^{\prime}$ and $6^{\prime} / \mathrm{m}^{\prime} \mathrm{m}$ 'm in the specimen ; these two groups differ by a translation along the $z$-direction (Fig. 2). Fortunately, point group $\overline{6}^{\prime} \mathrm{m} 2$ ' (which is considered unlikely for a coherent twin boundary) can be eliminated if the internal $2 \mathrm{~mm}$ symmetry of a $(2 \overline{2} 0)$ disc in Bragg position is

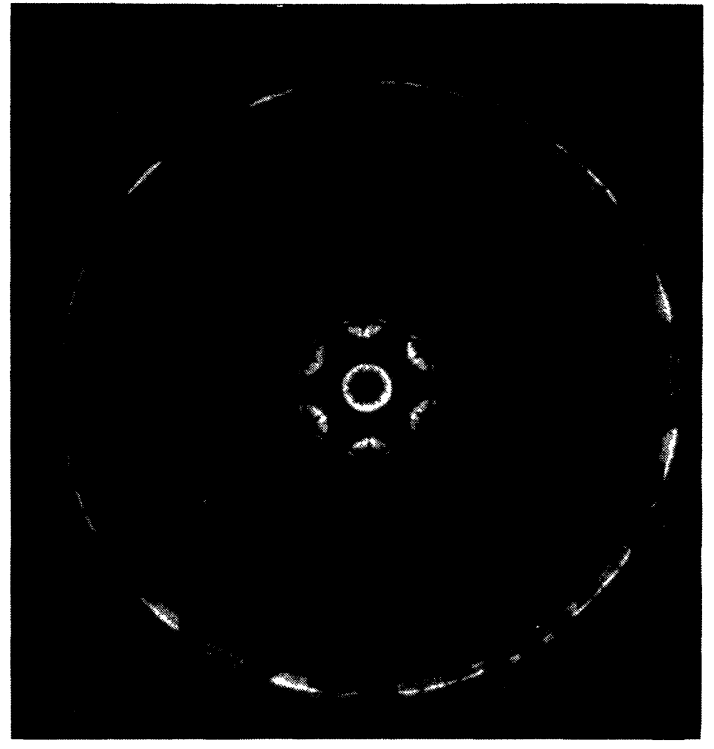

Fig. 4. - [111] zone-axis pattern of a twinned Au crystal. The $(000)$ disc in reverse contrast is shown enlarged in figure 6 (from Ref. [4]).

considered (Fig. 5). This symmetry can only be explained if the projection approximation in electron diffraction holds, and from table I of reference [4] it may be concluded that the correct dichromatic point group is $61 / \mathrm{m}^{\prime} \mathrm{m} / \mathrm{m}$, i.e. there is zero translation along the $z$-axis. Thus a unique point group determination is possible in this case.

A point of major concern in this method is the influence of a non-ideal boundary location in the specimen. In the theory developed so far the assumption that the boundary plane coincides with the specimen mid-plane is essential ; otherwise the reciprocity theorem coupled with a horizontal symmetry operation in the specimen cannot be applied. In order to investigate the effect of an asymmetric boundary location on the CBED pattern symmetry, a computer program has been developed to simulate

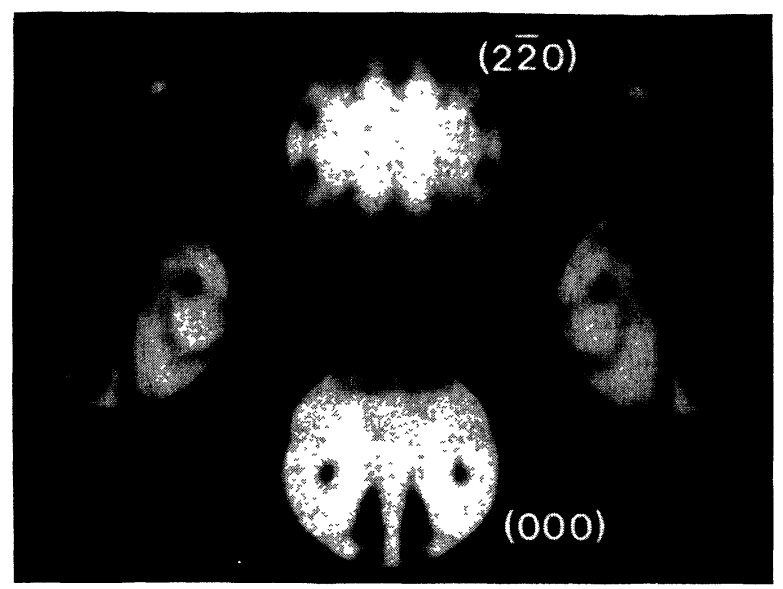

Fig. 5. - Internal $2 \mathrm{~mm}$ symmetry of a (220) disc in Bragg position, for the twinned Au crystal of figure 4 (from Ref. [4]). 


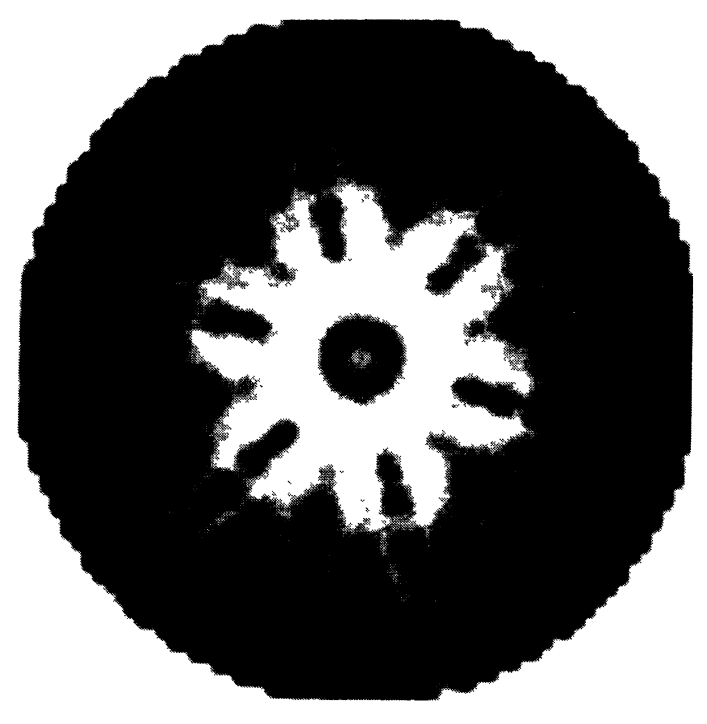

a)

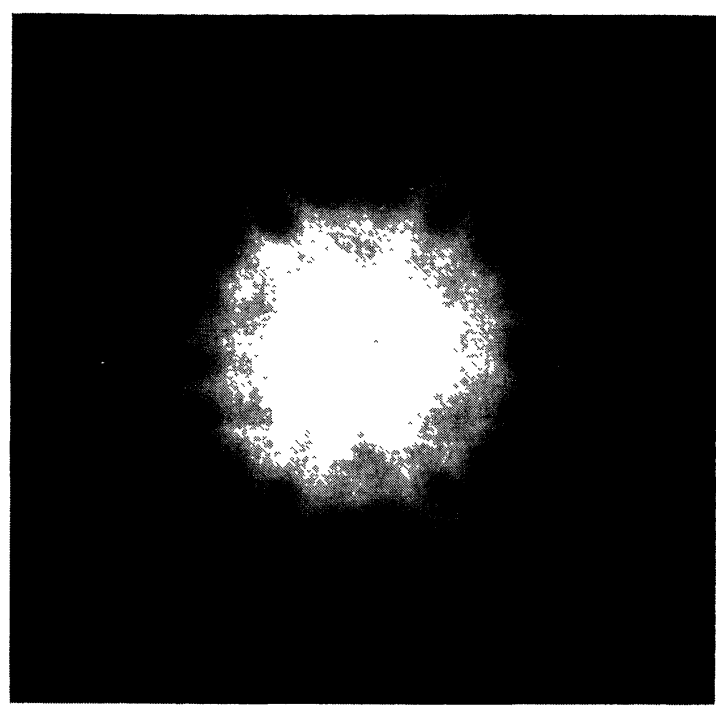

b)

Fig. 6. - Computer-simulated and experimental (000) disc for a twinned Au crystal in the same orientation as figure 4, shown in (a) and (b) respectively.

CBED patterns from bicrystals [12]. This program is based on the N-beam dynamical theory of electron diffraction ; the cone of incident beam directions is simulated by calculating diffracted intensities for a large number of plane incident waves. In the calculations it is assumed that the electron source is perfectly incoherent, i.e. the intensities of the various discs are constructed from the individual intensities diffracted from different incident beam directions. The program also may take absorption into account, which is especially important for relatively high atomic number materials. Figure 6 shows a simulated (000) disc for Au which should be compared with the corresponding (000) disc found experimentally. It can be seen that the agreement between both is very satisfactory. For the simulation a total specimen thickness of $80 \mathrm{~nm}$ has been assumed, an absorption parameter of 0.1 and the number of reflections taken into account is 55. It should be noted that the present level of agreement between experimént and simulation is only obtained as a result of taking into account the absorption. Without absorption the agreement is rather poor [13].

As mentioned, a major reason for performing simulations was to investigate the effect of a nonideal boundary location in a bicrystal specimen. This has been done in detail for a Si crystal containing an inclined twin boundary (Fig. 7). In figure 8 results for simulations of a Si bicrystal with a horizontal twin boundary located at various depths (expressed as a fraction $\xi$ of total specimen thickness) in the specimen are presented. It is seen that the $6 \mathrm{~mm}$ symmetry of the (000) disc occurring at the ideal boundary location $(\xi=0.5)$ is rapidly reduced to $3 \mathrm{~m}$ when the interface is shifted to an asymmetric position where, for example, $\xi=0.42$. It is interesting to compare these simulations with recently obtained results on an inclined twin boundary in a Si

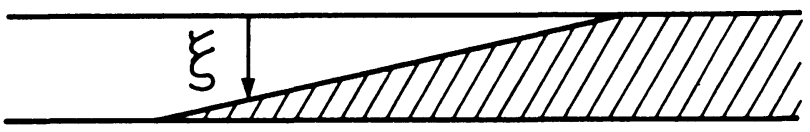

Fig. 7. - Schematic representation of an inclined grain boundary in a bicrystal. $\xi$ defines the boundary location (see text).

specimen, where CBED patterns could be taken at different values of $\xi$. Figure 9 presents some of the results ; as in the case of $\mathrm{Au}$ detailed agreement between experiment and simulation at $\xi=0.5$ could be obtained for the (000) disc assuming a specimen thickness of $200 \mathrm{~nm}$, taking into account 31 reflections and no absorption. Because of this agreement, a boundary location at $\xi \neq 0.5$ can best be determined by comparing the experimentally obtained intensity profiles with computations at different values of $\xi$ until agreement is obtained. It is also useful to remark that boundaries located at $\xi$ and $1-\xi$ give complementary intensities in the (000) disc, because of their symmetrical location relative to $\xi=0.5$ and the existence of the reciprocity theorem. These experiments on coherent twin boundaries show that detailed agreement between computer-generated and experimental CBED patterns from bicrystals can be obtained for low atomic number $(\mathrm{Si})$ as well as high atomic number $(\mathrm{Au})$ materials ; in the latter case it is essential to include absorption in the computations.

Finally we want to discuss some recent experiments on the symmetry of vertical coherent twin boundaries in Si [11]. As we have explained in section 2.2 a thin bicrystal with such a vertical boundary should be considered as a bicrystal band, reducing the number of possible point groups to 12 . For a Si bicrystal with a vertical (111) twin boundary 


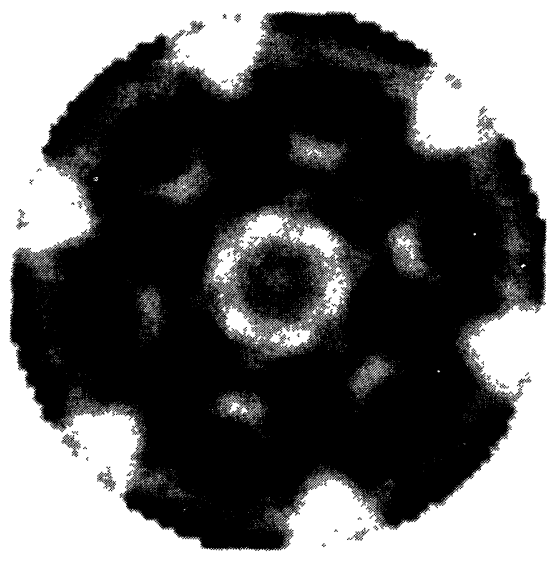

a)

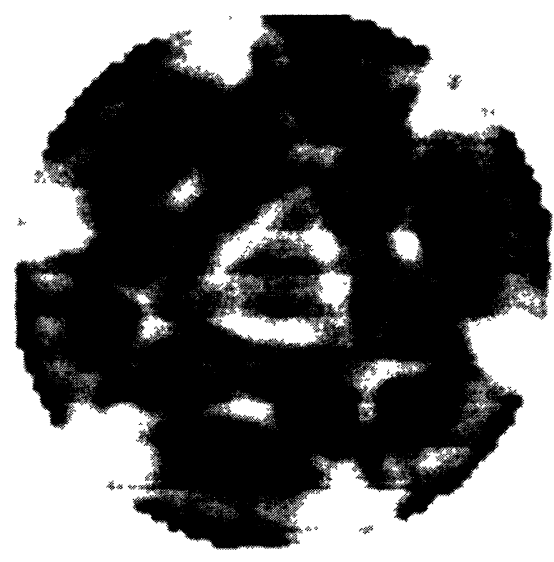

b)

Fig. 8. - Computer-simulated (000) discs of a [111] zone-axis pattern for a twinned Si crystal. The boundary plane is located at $\xi=0.5(\mathrm{a})$ and at $\xi=0.42(\mathrm{~b})$.

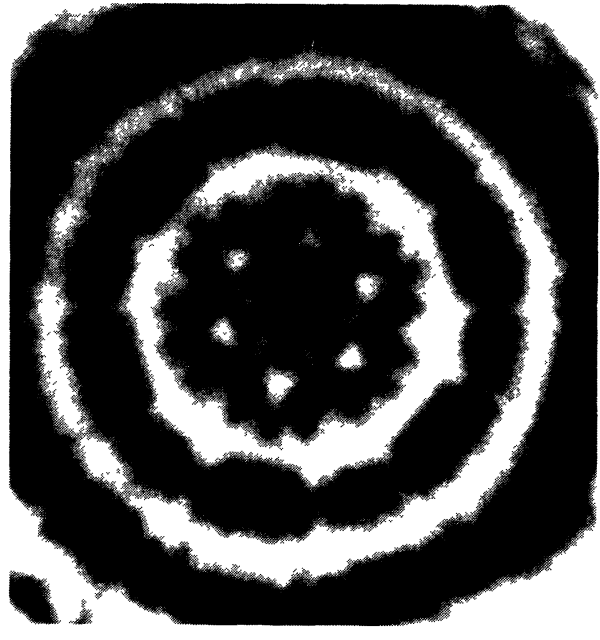

a)

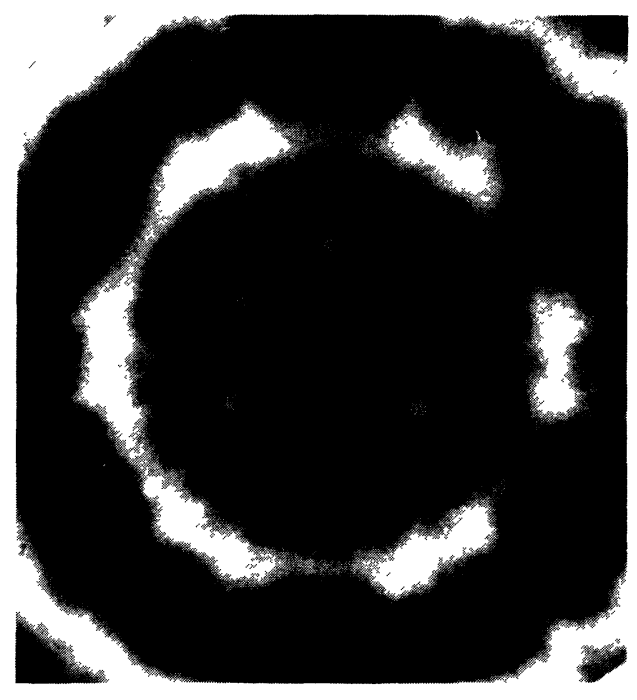

b)

Fig. 9. - Experimental (000) discs of a [111] zone-axis pattern from a twinned Si crystal with the boundary located at two different positions in the specimen.

the bicrystal point group is $\mathrm{m}^{\prime} \mathrm{m} 2^{\prime}$, leading to diffraction group $m 1_{\mathrm{R}}$ when the incident beam is parallel to the common [110] axis of the bicrystal (cf. Sect. 2.2). This diffraction group gives rise to $2 \mathrm{~mm}$ and $\mathrm{m}$ symmetry in the $(000)$ disc and whole pattern, respectively. Now, figure 10 shows a wide-angle zero beam disk (usually referred to as a Tanaka pattern) taken along the [110] axis of the Si bicrystal with the twin boundary running through the centre of the pattern. It is evident that the trace of the twin boundary plane is a mirror line in the pattern. However, a second mirror line, perpendicular to the first, is absent in figure 10 , so the symmetry of the (000) disc is only $\mathrm{m}$. Hence, a discrepancy exists between the expected $2 \mathrm{~mm}$ symmetry and the $\mathrm{m}$ symmetry found experimentally. It has been shown that this discrepancy is caused by the geometry of the incident beam in figure 10 , as illustrated schematically in figure 11 . The static convergent beam, centred as indicated in figure $11 \mathrm{a}$, is located such that the $\mathrm{m}$ (atrix) and $\mathrm{t}$ (win) crystals meeting at the boundary are illuminated by different sections of the cone of incident beams, i.e. these regions effectively see different incident beam directions. Consequently, the common horizontal $(2 \overline{2} 0)$ mirror planes in the specimen will not be detected by the electron beam, since application of the reciprocity theorem to figure 11a followed by the mirror operation of the (220) plane correlates opposite incident beam directions on the same side of the bicrystal specimen. If this explanation is correct, the full symmetry of the (000) disc should be present in a parallel rockingbeam geometry; see figure $11 \mathrm{~b}$. For this beam geometry a zone-axis pattern (ZAP) is formed, in 


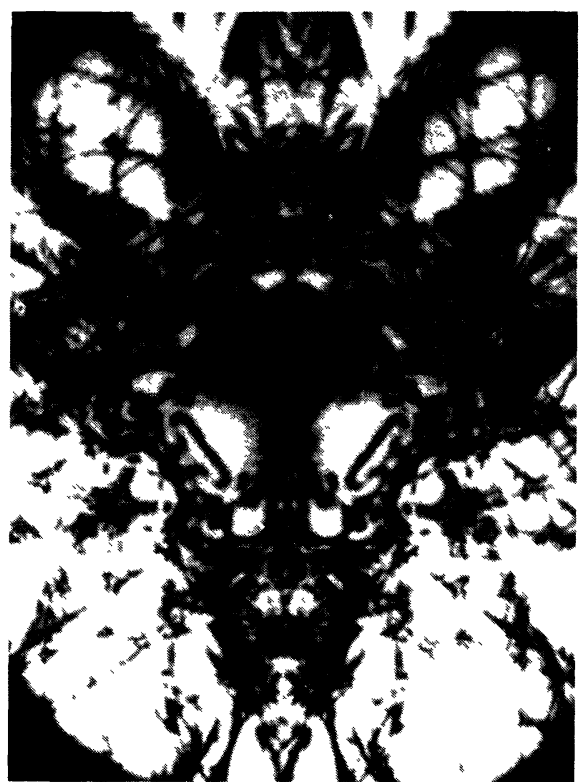

Fig. 10. - Tanaka pattern along [110] of a vertical coherent twin boundary in Si. The trace of the boundary plane runs vertically through the centre of the pattern (from Ref. [11]).

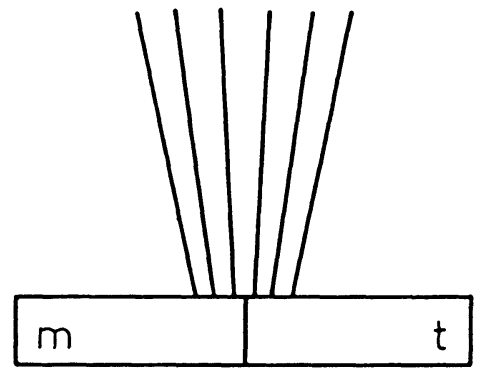

a)

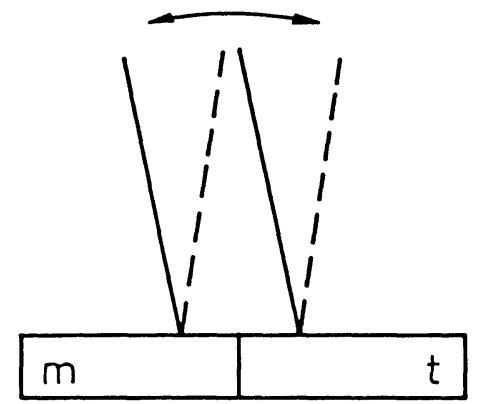

b)

Fig. 11. - Schematic diagram of the incident beam geometry for the case of a static beam (a) and a rocking beam (b).

which areas on either side of the boundary are sequentially illuminated by all incident beam directions, so the full symmetry should be revealed in such a pattern. To obtain such a pattern, the double rocking-beam technique developed by Eades [14] has been employed, and figure 12 shows a wide-

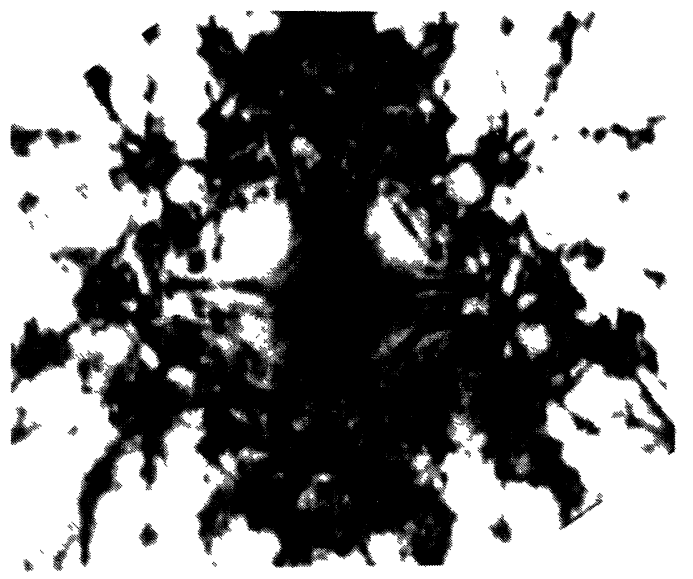

Fig. 12. - Double-rocking zone-axis pattern of an area similar to figure 10 , and in the same orientation as figure 10 (from Ref. [11]).

angle rocking-beam ZAP from an area similar to the one used to obtain figure 10 , confirming $2 \mathrm{~mm}$ symmetry of the pattern. It is thus seen that the symmetry of vertical grain boundaries can only be fully explored by the double rocking-beam technique, since for a static incident convergent beam any horizontal symmetry elements present in a specimen with a vertical boundary will be lost in the CBED pattern.

\section{Discussion.}

In this paper bicrystal symmetry determination has been restricted to the determination of point groups from an analysis of the symmetry of CBED patterns. However, as is well known, a full symmetry determination requires space group determination from a bicrystal. From the point of view of grain boundary structures this may be important, as specific rigidbody translations can lead to the occurrence of ordinary or coloured screw axes and/or glide planes in the bicrystal spatial group (in the terminology of Ref. [7]). The extension of point groups to spatial groups is most easily performed in the case of a vertical boundary. In this case there exist 31 possible spatial groups for bicrystal bands, consisting of 16 symmorphic and 15 non-symmorphic groups [7]. Since a bicrystal band has only 1-D translation symmetry, the 12 possible point groups of table IV are readily extended to the 16 symmorphic spatial groups. The remaining 15 non-symmorphic groups, containing ordinary or coloured screw axes and/or glide planes, can be determined from specific extinctions in the CBED pattern in a way analogous to that for a single crystal [15]. Although somewhat more complicated, a similar extension to spatial groups can be made for the point groups associated with a bicrystal layer (Tab. II).

In terms of relaxations of atoms in the boundary area it should be emphasized that the present method is restricted to the determination of those rigid-body translations that alter bicrystal symmetry. 
Local relaxation of atoms (shuffles) in the boundary area cannot be detected unless they change the symmetry. In this respect the method is comparable to Pond's method of determining translations from stacking-fault-like fringes. Both methods have their advantages and drawbacks [10] ; Pond's method is capable of determining translations very accurately in an inclined boundary, provided the proper diffraction conditions in TEM can be set up. For a vertical grain boundary on the other hand, CBED is an alternative to high-resolution EM (HREM). In the latter case only very thin specimens can be used, whereas CBED requires thicker specimens. HREM, in principle, is capable of giving accurate values for translations. However, only projected structures can be analysed in HREM, and hence no information on translations in the $z$-direction is obtained. Thus, it appears that the different methods mentioned here may give useful complementary information in boundary structure analysis.

In this paper the discussion has been limited to the structure of simple twin boundaries. Some experiments have also been performed on a $\Sigma=7$ (111) twist boundary in $\mathrm{Au}$ [5]. The dichromatic point group for this bicrystal with zero translation is $3 \mathrm{~m}^{\prime}$ and the corresponding bicrystal layer point group is $32^{\prime}$ (cf. Tab. II), leading to diffraction group $3 \mathrm{~m}_{\mathrm{R}}$. This group could be confirmed by a [111] ZAP taken from the bicrystal, although there was some local variation in CBED symmetry. This raises the question whether the CBED symmetry could be dependent on the probe size used in forming the diffraction pattern. Especially in higher $\Sigma$ boundaries, the boundary structure may consist of a regular array of domains, and such domains may or may not have structures related by a symmetry operation of the holosymmetric boundary structure $[7,16]$. Depending on the relative size of the probe and the domains, the CBED pattern symmetry may or may not correspond to the actual structure or symmetry of a single domain. In such cases it would therefore clearly be of interest to investigate whether the pattern symmetry is dependent on probe size. A similar situation may be found in low $\Sigma$ (e.g. twin) boundaries with a small deviation from the exact coincidence orientation, where a dislocation network accommodates the misfit.

\section{Conclusions.}

It has been shown in this paper that a correlation between bicrystal symmetry and CBED pattern symmetry enables quite useful information to be obtained on the state of translation existing at a boundary in a bicrystal. This correlation has been developed for both a horizontal interface in the specimen as well as for a vertical boundary. Since for a horizontal boundary the general theory only holds for an ideal boundary location (i.e. coinciding with the specimen mid-plane), a computer program has been constructed to generate CBED patterns from N-beam dynamical theory of electron diffraction. Employing this program a detailed comparison between computer-generated and experimental CBED patterns could be made for different boundary locations. It has been shown that a very satisfactory agreement between simulated and experimental patterns can be obtained for $\mathrm{Au}$ and $\mathrm{Si}$; in the former case it is essential to include absorption in the computations. As a consequence of this work CBED can now be considered as a valuable tool in grain boundary structure analysis, besides other established methods such as HREM and Pond's method employing TEM.

\section{Acknowledgments.}

The present review could not have been written without the cooperation of a number of past and present members of the group. In particular I wish to thank N. S. Blom, Mrs. R'Sue Caron and Drs. B. F. Buxton (GEC, Wembley, U.K.) and S.K.E. Forghany for their important contributions. The work is supported by the Netherlands Foundation for Chemical Research (SON) with financial aid from the Netherlands Organization for the Advancement of Pure Research (ZWO).

\section{References}

[1] Buxton, B. F., Eades, J. A., Steeds, J. W., RackHAM, G. M., Phil. Trans. R. Soc. London A 281 (1976) 171.

[2] Goodman, P., Electron Diffraction, 1927-77, edited by P. J. Dobson, J. B. Pendry and C. J. Humphreys (1978) p. 116.

[3] Goodman, P., Acta Crystallogr. A 40 (1984) 635.

[4] SchapinK, F. W., Forghany, S. K. E., BuXton, B. F., Acta Crystallogr. A 39 (1983) 805 ; Acta Crystallogr. A 40 (1984) 167.

[5] Buxton, B. F., Forghany, S. K. E., Schapink, F. W., Inst. Phys. Conf. Ser. 68 (1984) 59.

[6] Pond, R. C., Bollmann, W., Phil. Trans. R. Soc. London A 292 (1979) 449.

[7] Pond, R. C., Vlachavas, D. S., Proc. R. Soc. London A 386 (1983) 95.
[8] Gratias, D., Portier, R., J. Physique Coll. C6 43 (1982) 15.

[9] Kalonj, G., J. Physique Coll. C4 46 (1985) 249.

[10] SchapinK, F. W., Mertens, F. J. M., Scr. Metall. 15 (1981) 611.

[11] Schapink, ' F. W., Forghany, S. K. E., Caron, R. P., Philos. Mag. A 53 (1986) 717.

[12] Blom, N. S., SchapinK, F. W., J. Appl. Crystallogr. 18 (1985) 126.

[13] SChapinK, F. W., Blom, N. S., ForghaNy, S. K. E., J. Physique Coll. C4 46 (1985) 85.

[14] EADES, J. A., Ultramicroscopy 5 (1980) 71.

[15] SteEdS, J. W., VInCENT, R., J. Appl. Crystallogr. 16 (1983) 317.

[16] Vitek, V., Minonishi, Y., WANG, G. J., J. Physique Coll. C4 46 (1985) 171. 